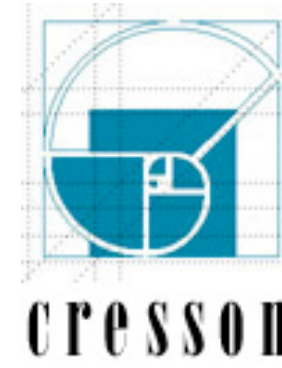

Jean-Paul Thibaud est chercheur CNRS au Laboratoire Cresson UMR 1563 Ambiances architecturales et urbaines, Ecole Nationale Supérieure d'Architecture de Grenoble / www.cresson.archi.fr

Pour citer ce document :

Thibaud, Jean-Paul. L'horizon des ambiances urbaines. Communications. $\mathrm{n}^{\circ}$ 73, 2002, pp. 185-201

\title{
L'horizon des ambiances urbaines
}

\author{
Jean-Paul Thibaud
}

Il arrive qu'un terme, d'un emploi courant et banal, acquiert une valeur scientifique et contribue à fonder un nouveau domaine d'investigation. Ce changement d'ordre de discours dans l'usage du mot n'est pas sans poser de problèmes théoriques et épistémologiques qui requièrent de toute évidence de nombreuses précautions. Qu'en est-il du terme " ambiance » en train de devenir un des enjeux de la recherche architecturale et urbaine ? Mettre la notion d'ambiance à l'épreuve de la pensée urbaine nécessite à la fois de tester sa portée heuristique et de faire œuvre de clarification. Autrement dit, comment et à quelles conditions une problématisation de la notion d'ambiance est-elle possible ? En quoi celle-ci nous permet-elle de renouveler nos façons de concevoir l'habiter ? Pour répondre à de telles questions, trois modes complémentaires peuvent être adoptés.

Premièrement, une lecture « exogène » rendra compte de la conjoncture scientifique dans laquelle s'inscrit le champ des ambiances. Il s'agira d'identifier les conditions de possibilité d'une approche sensible de la ville. D'une certaine manière, la notion d'ambiance émerge sur la base d'une convergence de résultats issus de champs de recherche habituellement dissociés.

Deuxièmement, une lecture « transverse » mettra cette notion en résonance avec d'autres termes qui lui sont apparentés. L'examen des complémentarités de voisinage contribuera à spécifier et positionner plus précisément la notion d'ambiance. Le chiasme dont elle relève la situera à la croisée d'une analyse esthétique et d'une analyse pragmatique de l'espace urbain.

Troisièmement, une lecture « endogène » fera état des problèmes théoriques qu'elle ne manque pas de poser. En mettant en œuvre une heuristique des paradoxes, il s'agira de dresser à grands traits l'intérêt et la portée opératoire d'une telle notion. C'est donc par approximations successives et variations de points de vue que se dégageront progressivement les lignes de force de ce champ d'investigation naissant. 


\section{Retour vers le concret}

Un des mouvements importants de la science contemporaine consiste à opérer un retour vers le concret ${ }^{1}$. En prenant appui sur la phénoménologie et sur le pragmatisme, de nombreux travaux proposent une alternative au dualisme cartésien en reconsidérant la place du corps dans notre façon d'appréhender le monde. La notion d'ambiance s'inscrit de plein droit dans cette perspective de l'embodiment ${ }^{2}$ pour laquelle nos catégories conceptuelles ne sont pas dissociables de notre activité sensori-motrice. A cet égard, quatre apports principaux intéressent plus directement la thématique des ambiances : la mise en évidence du pouvoir mobilisateur du lieu, la reconnaissance de la valeur articulatoire du geste, le dévoilement de l'implicite dans l'ordinaire des pratiques et l'ouverture de la perception à sa dimension affective.

\section{Le lieu revisité}

Nombre de travaux contemporains, issus de la philosophie, de l'architecture ou de la géographie humaine, revisitent la notion de lieu et cherchent à fonder ce que pourrait être une «topique ». En soulignant le caractère charnel et situé de l'expérience sensible, cette pensée du lieu se construit sur la base d'une critique de l'espace abstrait et objectif. Contrairement à l'espace conçu comme une étendue homogène, continue et divisible, sur le mode du partes extra partes, le lieu procède d'un investissement corporel indissociable de son pouvoir d'orientation et d'expression. Autrement dit, s'il n'y a pas de lieu sans corps et si le corps est d'emblée en prise avec le lieu, ce dernier ne peut pas être réduit à un pur contenant formel, ni à un simple système de coordonnées géométriques. A une théorie de l'espace sans qualités se substitue une approche du lieu incarné ${ }^{3}$. Une telle entreprise redonne toute son importance à l'argument de l'intensif et dévoile par là même une des caractéristiques de l'ambiance. Alors que l'espace est généralement appréhendé comme un ensemble de grandeurs extensives, le lieu permet de réintroduire l'idée de grandeurs intensives. Ainsi, l'espace est peuplé d'objets ou de parcelles que je peux discrétiser et dénombrer : un banc, une porte, un mur, un abribus, une

\footnotetext{
${ }^{1}$ Dès les années vingt, Alfred Whitehead développe l'idée de la "localisation fallacieuse du concret" (fallacy of misplaced concreteness) et nous met en garde de prendre l'abstrait pour le concret, au risque de se méprendre sur la nature de l'expérience vécue. Cf. Whitehead, A.N. Science and the Modern World. Cambridge, Cambridge University Press, 1926.

${ }^{2}$ Un bon panorama de cette position est présenté dans un ouvrage collectif récent : Weiss, $G$. \& Haber, H.F. (eds.) Perspectives on Embodiment. Londres, Routledge, 1996. Pour un examen plus approfondi de l'ancrage corporel de la cognition se reporter à deux ouvrages de toute première importance : Varela, F., Thompson, E. et Rosch, E. L'inscription corporelle de l'esprit : sciences cognitives et expérience humaine. Paris, Seuil, 1993 ; Johnson, M. The Body in the Mind. Chicago, The University of Chicago Press, 1987.

${ }^{3}$ Pour une lecture très documentée de l'histoire de la pensée du lieu, se reporter à l'ouvrage de synthèse de Edward Casey. Cf. Casey, E. The Fate of Place. A Philosophical History. Berkeley, University of California Press, 1998.
} 
cabine téléphonique, etc. De l'ordre du nombre et de la sommation, il relève de grandeurs extensives. Son unité procède d'une juxtaposition successive de parties distinctes. Par contre, le lieu engage des qualités sensibles plus ou moins prégnantes, qui peuvent croître ou décroître : luminosité, chaleur, rugosité, etc. De l'ordre du degré et du différentiel, il relève de grandeurs intensives ${ }^{4}$. Il en va alors de la manière dont le lieu se constitue comme unité immédiate et indivise ayant un caractère qui lui est propre. Sa cohérence interne ne résulte pas d'une sommation de parties juxtaposées mais plutôt d'un geste qui saisit d'emblée une globalité. De ce point de vue, si le lieu convoque bien une mise en forme du cadre bâti, il procède d'un rythme qui lui donne sa véritable consistance ${ }^{5}$. Réalité d'ordre rythmique et énergétique, le lieu relève moins d'une logique de l'inclusion que d'une logique de l'exposition. En mettant le corps en mouvement, en l'affectant et en le saisissant, il fait montre d'une puissance d'imprégnation qui ne laisse pas intact celui qui le traverse ${ }^{6}$. Tout est alors question de forces et de degrés, de tensions et de détentes, d'allures et de postures. Bref, le lieu ne se réduit en aucun cas à une enveloppe désaffectée et inopérante, il habite le corps en même temps qu'il se laisse habiter par lui. Pour finir, penser l'espace selon la catégorie de l'intensif permet de faire valoir l'efficace sensori-moteur de l'environnement construit en redonnant sa juste place aux phénomènes rythmiques qui engagent notre rapport au monde ambiant.

\section{Le geste à l'œuvre}

L'affinité étroite du lieu et du corps nous ramène tout naturellement à la question du geste. Affirmer que « l'être dans le monde se manifeste par des gestes ${ }^{7}$ conduit à reconsidérer le rôle central de la motricité dans nos façons d'agir. Il s'agit dès lors de se situer en-deçà de l'expérience

\footnotetext{
${ }^{4}$ La distinction entre grandeur extensive et grandeur intensive est développée en particulier par Emmanuel Kant: "Tout réel d'une même qualité a le degré (de résistance ou de pesanteur) de cette qualité, degré qui, sans que diminue la grandeur extensive ou le nombre, peut décroître jusqu'à l'infini avant que cette qualité disparaisse dans le vide et s'évanouisse. Ainsi, une dilatation qui remplit un espace, par exemple la chaleur, peut sans laisser le moins du monde vide la plus petite portion de cet espace, décroître en degrés jusqu'à l'infini, sans néanmoins cesser d'emplir l'espace, avec ces degrés plus petits, tout aussi bien qu'un autre phénomène avec de plus grands ». Cf. Kant, E. [1781] Critique de la raison pure. Paris, Gallimard, 1980.

${ }^{5}$ Henri Maldiney exprime admirablement cette idée dans la définition qu'il donne du lieu : « Une architecture n'est pas un assemblage d'arcs, de voûtes, de colonnes, de pleins et de vides, elle est un rythme unique qui les traverse toutes et à cet égard, on peut dire qu'elle transcende les structures formelles de l'espace géométrique. L'espace transformel impliqué dans le rythme d'une architecture constitue un lieu ». Cf. Maldiney, H. Topos-Logos-Aisthèsis. in Le sens du lieu. sous la direction de Michel Mangematin, Philippe Nys et Chris Younès, Bruxelles, éditions Ousia, 1996, pp. 13-34.

${ }^{6}$ L'argument d'une topique comme pensée injonctive est développé en détail dans le bel ouvrage de Jean-Marc Ghitti. Cf. Ghitti, J.M. La parole et le lieu. topique de l'inspiration. Paris, Minuit, 1998.

${ }^{7}$ Telle est la proposition formulée et défendue par Vilem Flusser. Cf. Flusser, V. Les Gestes. Paris, D’Arts éditeur - HC, 1999.
} 
consciente et de faire valoir le plan pré-réflexif des conduites quotidiennes. Remarquons tout d'abord que le monde nous est donné non comme une pure extériorité saisissable selon une position de surplomb mais comme un ensemble de situations concrètes mettant à l'épreuve nos aptitudes et dispositions à agir. De ce point de vue, recourir à la notion de geste permet de dépasser les apories du modèle de l'action rationnelle en abordant sous un jour nouveau le double problème de l'intention et de l'expression. D'une part, à une conception propositionnelle et téléologique de l'intentionnalité se substitue une conception motrice et écologique ${ }^{8}$. L'action se structure moins sur la base d'une représentation de fins fixées à l'avance que de gestes qui s'ajustent progressivement en fonction des ressources et des contraintes de l'environnement. Si certaines situations critiques ou problématiques altèrent l'action routinière et nous amènent parfois à en prendre conscience, la plupart des gestes quotidiens relèvent d'un «affairement absorbé » 9 consistant à répondre aux sollicitations de la situation indépendamment de toute représentation. D'autre part, si le geste renvoie bien au monde de l'action, il ne se confond pas pour autant avec lui. En tant que support de l'action $^{10}$, il se prête à des variations et des modulations qui expriment à même le corps les tonalités affectives du moment. Comme nous l'apprend la danse, la capacité du geste à épouser le terrain, à décliner des styles de motricité et des qualités de mouvements participe des diverses manières d'être dans un milieu. Autrement dit, le geste n'est ni seulement de l'ordre de la simple fonctionnalité, ni de la pure expressivité, il articule dans un même temps la dimension pragmatique et la dimension esthétique de l'habiter ${ }^{11}$. Prendre au sérieux les gestes du quotidien permet ainsi de mettre l'accent sur la plasticité du corps en reconnaissant l'existence de schèmes d'expérience préconceptuels et prélinguistiques.

\section{L'ordinaire en commun}

Les thématiques du lieu et du geste s'inscrivent dans un questionnement plus général portant sur l'ordinaire de l'expérience urbaine. Il s'agit dès lors de problématiser ce qui va de soi dans la vie de tous les jours et d'interroger ce qui est donné habituellement pour évident. Autrement dit, le monde quotidien nous est familier dans la mesure où l'ensemble de règles et de

\footnotetext{
${ }^{8}$ Sur l'idée d'intentionnalité motrice ou opérante, se reporter aux travaux précurseurs et toujours actuels de Maurice Merleau-Ponty. Cf. Merleau-Ponty, M. Phénoménologie de la perception. Paris, Gallimard, 1945.

${ }_{9}$ Hubert Dreyfus développe l'idée d' « affairement absorbé » (absorbed coping) pour rendre compte du rapport corporel immédiat que nous entretenons avec les situations de tous les jours. Cf. Dreyfus, H. Being-in-the-World: a Commentary on Being and Time. Cambridge, MIT Press, 1991.

${ }^{10}$ Le terme « geste » vient du latin gestus construit sur la racine de gero, gerere qui signifie à la fois faire et porter. Pour Giorgio Agamben, le geste remet en cause la distinction entre fins et moyens en rendant visible « un moyen comme tel ». Cf. Agamben, G. Moyens sans fins. Paris, éditions Payot, 1995.

${ }^{11}$ Cette proposition est à la base du remarquable essai de Michel Guérin. Cf. Guérin, M. Philosophie du geste. Arles, Actes Sud, 1995.
} 
procédures, d'habitudes et d'attentes sur lesquelles nous nous appuyons constamment est tenu pour acquis une fois pour toute. Rendre compte du caractère ordinaire de la vie en commun suppose d'éclairer sous un jour nouveau le problème de la compréhension d'arrière-fond. Pour ce faire, il faut de se départir d'une approche par trop herméneutique et se situer « sous l'interprétation $\gg^{12}$. En effet, l'activité interprétative à laquelle nous nous livrons parfois pour faire face aux situations est sous-tendue par des savoirfaire corporels exempts de toute délibération. Adopter un rythme commun, enchaîner au moment opportun, maintenir la bonne distance, ajuster sa posture à bon escient sont autant d'opérations implicites et néanmoins fondamentales de l'être-ensemble ${ }^{13}$. L'existence et l'actualisation de telles procédures constituent un fonds commun à toute interaction sociale, condition sine qua non de la sociabilité en public. Définir de la sorte la compréhension conduit à problématiser le lien social en terme d'intercorporéité, de chorégraphie tacite à laquelle nous prenons tous part mais qui se joue aussi à notre insu. Mais encore, si l'on parle d'arrière-fond c'est parce que l'on ne peut facilement dissocier cette communication entre les corps de la culture dans laquelle elle est enchâssée. De ce point de vue, si le corps est bien le support par excellence de la compréhension, il permet de sédimenter et de reconduire l'ensemble des composantes d'une forme de vie, qu'il s'agisse des artefacts techniques et dispositifs matériels, des règles et codes en vigueur, des activités et pratiques du moment, des manières de sentir et de s'exprimer ${ }^{14}$. Questionner ainsi l'ordinaire permet de mettre à l'épreuve le sentiment de familiarité comme composante fondamentale de l'habiter en révélant l'existence d'une base commune nous reliant les uns aux autres.

\section{Le sensible qualifié}

Plus directement affiliée à la notion d'ambiance, la thématique du sensible pose à nouveaux frais la question de la perception. Si l'expérience vécue convoque un moment gnosique de l'ordre du percevoir, elle engage

\footnotetext{
12 Titre d'un ouvrage de Richard Shusterman qui montre que l'on ne peut réduire la compréhension à un niveau exclusivement interprétatif. Cf. Shusterman, R. Sous l'interprétation. traduit de l'anglais par Jean-Pierre Cometti, Combas, éditions de l'Eclat, 1994.

${ }^{13}$ Comme l'analyse Charles Taylor, si cette compréhension d'arrière-fond est dans une large mesure incorporée et incarnée dans nos façons de bouger, elle ne revient pas pour autant à développer un individualisme méthodologique ou à soutenir une perspective egologique de l'agir humain. Cf. Taylor, C. Suivre une règle. Critique. $n^{\circ}$ 579-580, août-septembre 1995, pp. 554-572.

${ }^{14}$ Elizabeth Behnke développe l'idée de « gestes fantômes » (ghost gestures) pour rendre compte de la dimension anonyme et pré-individuelle des schèmes moteurs qui structurent nos conduites quotidiennes. Cf. Behnke, E. Gost Gestures: Phenomenological Investigations of Bodily Micromovements and Their Intercorporeal Implications. Human Studies. Vol. 20, $\mathrm{n}^{\circ} 2$, 1997, pp. 181-201.
} 
également un moment pathique de l'ordre du sentir ${ }^{15}$. Une telle distinction permet de reconnaître l'irréductibilité de la perception à sa dimension cognitive en faisant valoir un mode de communication immédiat avec le monde. Plutôt que de le catégoriser, il s'agit ici de l'éprouver et de le ressentir. De ce point de vue, le monde ambiant ne relève pas seulement d'un monde d'objets clairement identifiables, il convoque des qualités qui spécifient des modes de présence et des manières d'apparaître. Cette attention portée à la dimension phénoménale des situations engage trois plans constitutifs du sensible : la motricité, l'affectivité et la diffusivité. Les qualités sensibles ne sont pas des états fermés sur eux-mêmes et indépendants de l'activité du sujet percevant, elles sollicitent des conduites motrices qui les fait paraître en retour ${ }^{16}$. Autrement dit, sentir et se mouvoir constituent deux versants indissociables du monde ambiant. Ce mouvement de phénoménalisation qui introduit le bougé au sein même du sensible n'est pas pour autant désaffecté. Bien au contraire, les qualités sensibles prennent corps à partir du moment où se déploie le champ de l'affectivité. Tel phénomène se dote d'une qualité dans la mesure où il est perçu comme apaisant ou effrayant, enjoué ou accablant, etc. Loin d'être un simple trouble centré sur le sujet, l'émotion procède d'un mouvement d'ouverture au monde permettant de le saisir d'une certaine manière ${ }^{17}$. Mais encore, si le sensible s'éprouve c'est avant tout de façon diffuse, en termes de tonalités affectives. D'une part, une tonalité affective colore la globalité de la situation présente en lui conférant une certaine physionomie. Cette dimension atmosphérique de l'émotion ne se subordonne ni à l'état psychique d'un sujet, ni à tel objet particulier de l'environnement. Elle est indistinctement sentiment du moi et du monde. D'autre part, une tonalité affective ne s'impose pas nécessairement de façon soudaine et violente. Elle procède en général par petites touches, par imprégnation légère faite d'infimes variations ${ }^{18}$. De ce point de vue, nul n'est besoin de la remarquer ou d'en prendre véritablement conscience pour qu'elle imprime sa marque sur nos faits et gestes quotidiens. Les tonalités affectives sont donc diffuses parce que non localisables et infra-conscientes.

Ces divers champs de questionnement intéressent la notion d'ambiance en cela qu'ils fournissent des « leviers conceptuels » permettant de formuler quelques propositions de base.

\footnotetext{
${ }^{15}$ La distinction entre «moment gnosique » et «moment pathique » est établie par Erwin Straus dans un ouvrage qui a fait date et qui n'a été traduit en français que récemment. Cf. Straus, E. [1935] Du Sens des Sens. traduit de l'allemand par G. Thines et J.P. Legrand, Grenoble, Jérôme Millon, 1989.

${ }^{16}$ Sur l'idée d'un «paraître moteur ", se reporter à : Barbaras, R. Motricité et phénoménalité chez le dernier Merleau-Ponty. in Merleau-Ponty. phénoménologie et expériences. textes réunis par Marc Richir et Etienne Tassin, Grenoble, Jérôme Millon, 1992, pp. 27-42.

${ }^{17}$ Rappelons que dans son acception étymologique, l'émotion vient directement du latin exmovere qui signifie «mouvoir hors de ". Pour un développement de ce rapport étroit entre mobilité et émotion, se reporter à : Mazis, G. Emotion and Embodiment: Fragile Ontology. New York, Peter Lang, 1993.

${ }^{18}$ On reconnaîtra ici l'héritage leibnizien des " petites perceptions ». Pour un exposé de l'idée d'atmosphère selon cette perspective, se reporter à : Gil, J. Les petites perceptions. Chimères. $\mathrm{n}^{\circ} 39$, été 2000, pp. 9-20.
} 


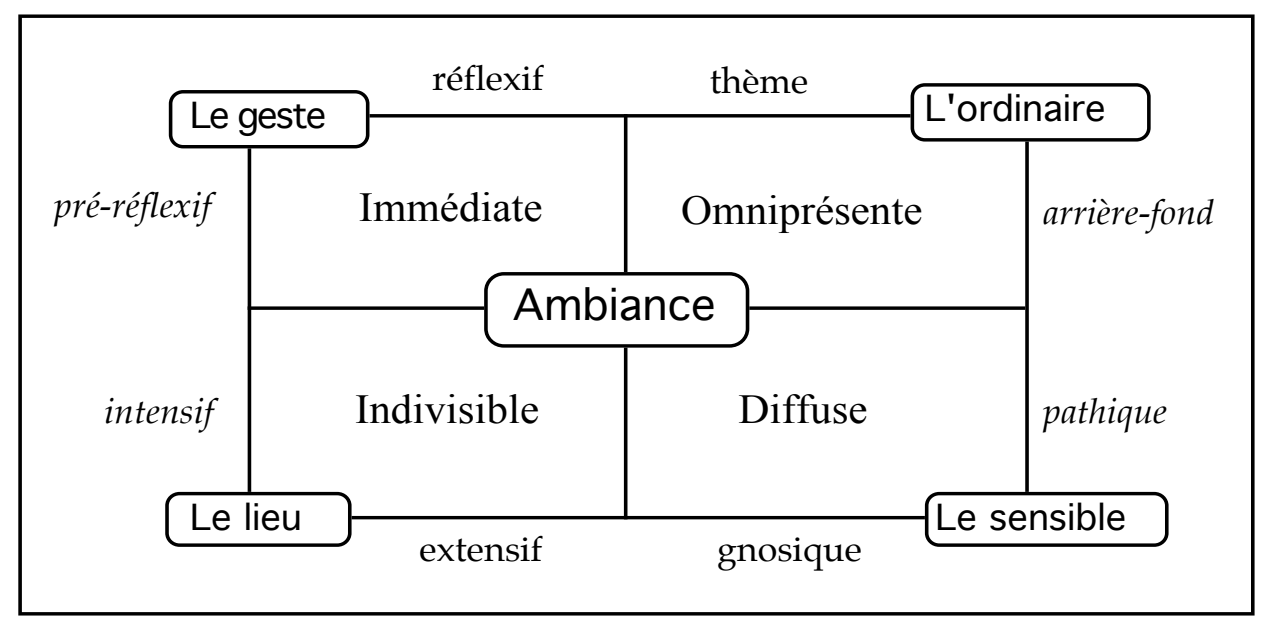

Premièrement, l'ambiance est indivisible. Admettre qu'on ne peut la saisir que dans sa globalité nécessite de réintroduire la catégorie de l'intensif en l'articulant à celle de l'extensif. De ce point de vue, une ambiance peut être caractérisée selon son degré de prégnance. Deuxièmement, l'ambiance est immédiate. Convenir qu'elle procède d'une mobilisation corporelle nécessite de réintroduire le versant pré-réflexif de l'expérience en l'articulant à son versant réflexif. De ce point de vue, une ambiance se spécifie par le style de motricité qu'elle convoque. Troisièmement, l'ambiance est omniprésente. Affirmer qu'on ne peut en rendre compte que de l'intérieur nécessite de réintroduire l'idée d'arrière-fond en l'articulant à celle de premier plan thématique. De ce point de vue, une ambiance se singularise par les dynamiques de variations auxquelles elle se prête. Quatrièmement, l'ambiance est diffuse. Déclarer qu'elle engage de l'affect nécessite de réintroduire la pente pathique de la perception en l'articulant à sa pente cognitive. De ce point de vue, une ambiance s'individue à diverses échelles qu’il s'agit précisément d'identifier.

\section{Le chiasme des ambiances}

Une deuxième façon de préciser la notion d'ambiance est de mettre en évidence ce qui la définit en propre. Plus précisément, il s'agit de caractériser le mode de positionnement qu'elle implique par rapport à des notions voisines. Travailler sur les marges et les écarts qu'elle entretient avec les notions de «paysage » et de «situation » ressortit d'une approche pragmatique encore à l'état d'ébauche. Ainsi se dégage le chiasme des ambiances : les théories du paysage rendent compte des qualités sensibles du monde urbain mais restent la plupart du temps inopérantes pour penser son efficace pratique ; à l'inverse, les théories de l'action mettent l'accent sur le caractère pratique de la perception mais demeurent insensibles à son régime pathique. 


\section{Le paysage en pratique}

L'intérêt des travaux actuels sur le paysage est d'offrir un cadre théorique permettant de penser le rapport sensible au monde environnant. En ne se limitant plus à l'analyse du paysage «naturel» et à un travail sur la représentation picturale, nombre de démarches s'interrogent désormais sur la dimension esthétique de l'expérience urbaine contemporaine. S'il existe de nombreuses façons de concevoir le paysage, la plupart d'entre elles reconnaissent son versant affectif et émotionnel. A cet égard, l'organisation perceptive qui préside à la constitution du paysage ne se réduit pas à une activité purement cognitive mais engage aussi le plan du sentir ${ }^{19}$. Cet intérêt porté au versant pathique de la perception et aux tonalités affectives du monde rapproche la notion de paysage de celle d'ambiance. Toutefois, la plupart des théories esthétiques du paysage tendent à négliger la portée pratique de la perception située. Tout se passe comme si l'expérience du paysage neutralisait nécessairement le regard en action. Inscrit dans un espace de représentation, le paysage semble ainsi reposer sur une attitude essentiellement contemplative, s'organiser à partir d'un point de vue fixe et unique, supposer une nécessaire mise à distance du sujet percevant.

Pourtant, certains travaux actuels proposent des échappées salutaires à cette conception dominante et dressent en quelque sorte des lignes de fuite mettant à l'épreuve le rapport entre agir et sentir. C'est souvent à partir d'un décentrement par rapport à l'esthétique paysagère moderne que de telles perspectives se sont développées. Ainsi, l'intérêt porté à la culture japonaise et à sa conception du paysage permet de réintroduire la mobilité et la sociabilité dans l'esthétique paysagère ${ }^{20}$. A une vision du monde fondée sur l'idée de sujet se substitue une approche dynamique qui introduit la participation du public comme composante à part entière du paysage. Une autre façon d'interroger les catégories convenues du paysage moderne consiste à les mettre à l'épreuve d'une autre modalité sensible que le visuel. Ainsi, quand on se demande ce qu'est un paysage au niveau sonore, d'autres critères de définition ne manquent pas d'émerger ${ }^{21}$. La prise en compte de la

\footnotetext{
${ }^{19}$ Bien que fortement connotée par l'usage qu'en a fait le romantisme, l'idée de Stimmung exprime bien le rapport intrinsèque entre l'unité perceptible qui instaure le paysage et le sentiment constitutif de ce dernier. Cf. Simmel, G. [1913] Philosophie du paysage. in La tragédie de la culture. Petite Bibliothèque Rivages, Paris, 1988. Terme inexistant en français, traduit souvent par «atmosphère » ou «ambiance ", la Stimmung désigne la tonalité d'ensemble d'une portion du monde visible et assure par là-même la cohérence interne d'éléments jugés autrement disparates et hétérogènes.

${ }^{20}$ Les notions de «point de perception mobile " faisant valoir l'expérience du paysage en mouvement, de «conjonction souple » révélant l'existence de règles de bienséance et de mitate indiquant l'usage de références qui instituent des façons de voir partagées relativisent considérablement les catégories héritées de la philosophie occidentale. Outre les nombreux travaux d'Augustin Berque portant sur le paysage japonais, se reporter aussi au bel article de Yoshio Nakamura. Cf. Nakamura, Y. Tradition paysagère et post-modernité au Japon. in Le débat. $n^{\circ} 65$, mai-août 1995, pp. 75-87.

${ }^{21}$ Pour Jean-François Augoyard, l'écoute paysagère du monde sonore repose davantage sur des combinaisons variables de la figure et du fond que sur un rapport stable et durable
} 
dimension temporelle et de la force expressive du son conduit alors à privilégier le sentiment d'immersion du sujet percevant sur sa mise en retrait supposée. Enfin, certaines approches poétiques introduisent la place du corps dans l'expérience et l'invention du paysage. D'une certaine manière, il s'agit de redécouvrir l'immédiateté du rapport à la matière sensible en s'émancipant autant que faire se peut du monde de la représentation. L'attention portée au rythme, à l'intonation et à la mélodie d'un poème révèle alors comment le corps se charge d'affects et se met à disposition du paysage $^{22}$.

De telles perspectives amorcent et annoncent la possibilité d'un retournement de la pensée du paysage : le mouvement tend à se substituer au point fixe, le social à l'individuel, le corporel au visuel, l'immersion à la distanciation. Alors que le paysage est appréhendé habituellement sur le mode de la vision contemplative, ces apports récents profilent en filigrane le plan de l'action et ébauchent une approche du « paysage en pratique ${ }^{23}$.

\section{Le sentiment de la situation}

Les théories de l'action, issues en particulier de la sociologie anglo-saxonne (ethnométhodologie, cognition distribuée, interactionnisme, ethnographie de la communication, etc.), se développent actuellement sur la base d'un double mouvement : attention croissante portée à l'ordinaire de la perception et exploration des pratiques de la rue comme domaine d'investigation. En cherchant à comprendre comment les situations de tous les jours se déroulent, s'ordonnent et se dotent de sens, ces démarches proposent un modèle praxéologique de la perception ${ }^{24}$. Dès lors que l'on se propose de décrire la vie sociale à même ses accomplissements pratiques se pose la question du rapport entre agir et percevoir. Ces deux catégories constituent deux pentes complémentaires et indissociables de l'activité, si bien que toute théorie de l'action pratique engage inévitablement une conception de la perception située. Par contre, en traitant prioritairement de la dimension pratique de la perception, cette posture tend à négliger son versant pathique. D'une certaine manière, les théories de l'action tendent à neutraliser les dimensions phénoménales et qualitatives du monde environnant.

\footnotetext{
(métabole), engage la disjonction plutôt que la continuité et l'homogénéité spatiales (discrétisation), remet en cause le dualisme de l'objet et du sujet au profit d'une dynamique du proche et du lointain. Cf. Augoyard, J.F. La vue est-elle souveraine dans l'esthétique paysagère ? in Le débat. $\mathrm{n}^{\circ}$ 65, mai-août 1995, pp. 51-59.

${ }^{22}$ On reconnaîtra, entre autres, le propos du bel ouvrage de Michel Collot. Cf. Collot La Matière-émotion. PUF, Paris, 1997.

${ }^{23}$ Isaac Joseph a proposé un développement argumenté de cette question. Cf. Joseph, I. Paysages urbains, choses publiques. in Les carnets du paysage. $\mathrm{n}^{\circ} 1$, printemps 1998 , pp. 70 88

${ }^{24}$ Pour un exposé particulièrement explicite de cette position, se reporter à : Coulter, J. \& Parsons, E.D. The Praxiology of Perception: Visual Orientations and Practical Action. Inquiry. An Interdisciplinary Journal of Philosophy. Vol. 33, n 3, 1990, pp. 251-272.
} 
Certains travaux ouvrent pourtant différentes pistes qui pourraient aider à dépasser cette aporie. Ainsi, l'intérêt croissant accordé à la place des émotions dans le déroulement des échanges quotidiens vise à dépasser une pensée par trop intellectualiste de l'action ${ }^{25}$. De ce point de vue, la dichotomie traditionnelle entre action et passion semble belle et bien remise en cause. Pour aussi intéressante qu'elle soit, une telle tentative se situe sur un plan essentiellement cognitif et n'accorde que peu de crédit à la question de la sensibilité. D'autres recherches, en lien avec les résultats les plus récents des neurosciences, proposent de penser l'action en terme de motricitée ${ }^{26}$. En s'intéressant aux schémas moteurs qui sous-tendent l'action, cette démarche tente de saisir le lien entre le conatif et le cognitif. Si revenir à ce niveau élémentaire de l'action aide à penser la dimension affective et la plasticité du geste, c'est au prix d'une nécessaire réduction de la complexité des situations concrètes. A cet égard, la micro-écologie des activités sociales propose une échelle d'analyse sans doute très adaptée à l'expérience de tous les jours ${ }^{27}$. Non seulement elle permet de rendre compte du cadre matériel et perceptif à partir duquel s'organise les interactions sociales, mais elle aide à distinguer les situations en fonction de leur valeur affective. On parle alors de situation «tendue » ou « relâchée », " embarrassante » ou " apaisée », " tranquille » ou " fatale », etc. Par contre, en accordant le primat à l'expression et à l'activité humaines, cette approche tend à négliger les composantes sensibles de l'environnement construit. Tout se passe comme si les qualités sonores, lumineuses, olfactives ou thermiques du milieu ambiant ne jouaient que peu de rôle dans l'émergence des tonalités affectives du moment ${ }^{28}$.

Ces quelques références montrent ainsi que certaines démarches issues des théories de l'action ne sont pas étrangères à la problématique des ambiances. En posant la question de l'émotion, de la motricité ou du style des interactions, elles se rapprochent, chacune à leur manière, de ce que l'on pourrait appeler sommairement le « sentiment de la situation ».

Au terme de ce second parcours, on peut situer l'ambiance au croisement d'une esthétique et d'une pragmatique de l'espace urbain. L'hypothèse sousjacente est que la notion d'ambiance permet d'intégrer ce double versant de l'expérience de manière à penser à nouveaux frais le rapport entre agir et sentir. Plus précisément, ce chiasme des ambiances consiste à articuler le paysage en pratique et le sentiment de la situation.

\footnotetext{
${ }^{25}$ Se reporter aux travaux de Patricia Paperman, et parmi eux à un recueil d'articles qu'elle a co-dirigé avec Ruwen Ogien. Cf. Paperman, P. et Ogien, R. [dir.) Raisons Pratiques. « La couleur des pensées. Sentiments, émotions, intentions ». $n^{\circ} 6$, éditions de l'EHESS, Paris, 1995.

${ }^{26}$ Dans cette perspective pluri-disciplinaire, citons en particulier l'ouvrage de Pierre Livet. Cf. Livet, P. La communauté virtuelle. Combas, L'Eclat, 1994.

${ }^{27}$ Nous pensons en particulier à l'œuvre magistrale de Erving Goffman.

${ }^{28}$ Sans doute faudrait il revenir alors à l'argument de John Dewey selon lequel « une situation est un tout en vertu de sa qualité diffuse immédiatement perceptible ». Cf. Dewey, J. [1938] Logique. La théorie de l'enquête. Paris, P.U.F., 1993.
} 


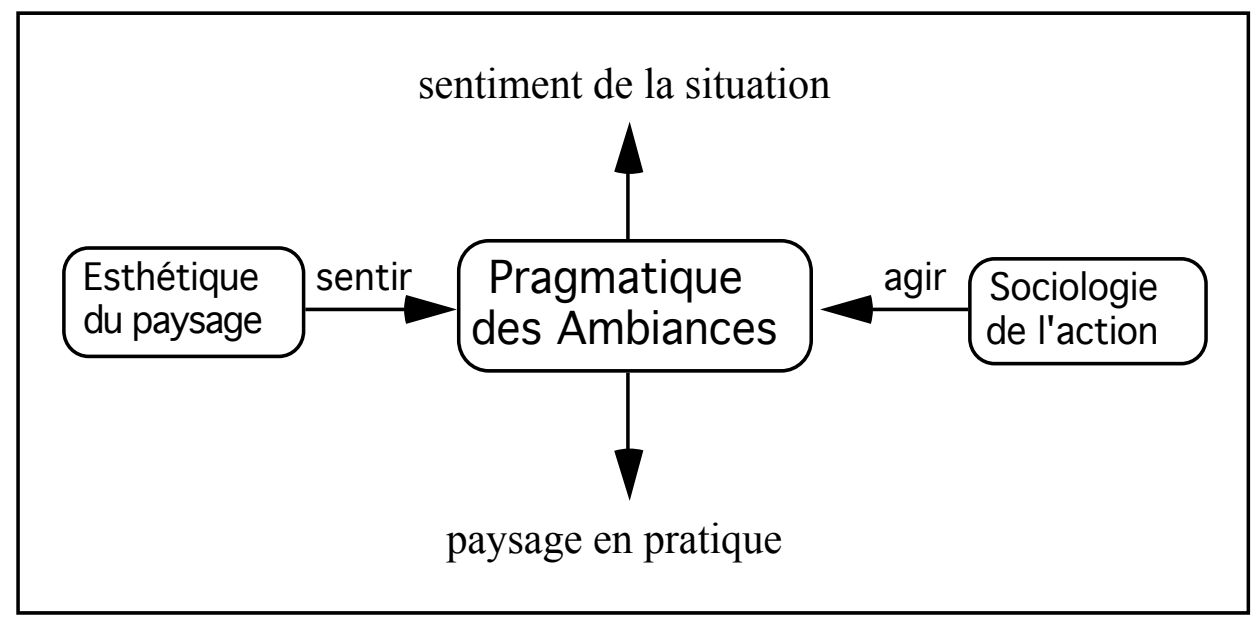

Pour ce faire, un tel projet nécessite de reconsidérer notre manière même de concevoir l'esthétique et le pragmatisme. D'une part, plutôt que de penser l'esthétique en terme de jugement de goût, il s'agit de la poser en terme d'aisthesis, c'est-à-dire comme une discipline qui s'intéresse à la sensorialité elle-même, à l'expérience sensible quelle qu'elle soit. De ce point de vue, c'est la relation entre des qualités environnementales et la sensibilité humaine qui doit être questionnée ${ }^{29}$. D'autre part, si le pragmatisme traite effectivement de l'agir humain, il ne doit pas pour autant postuler a priori une «relation activiste avec le monde ». Cette version du pragmatisme doit permettre au contraire d'intégrer la passivité, la sensibilité et la réceptivité au sein même de l'action $^{30}$. Pour résumer, la notion d'ambiance conduit à repenser la relation entre l'organisme humain et son environnement en procédant à une « sensibilisation de la pratique ».

\section{Sensibilisation de l'habiter}

Une dernière façon de cerner la notion d'ambiance est de la travailler de l'intérieur, en s'appuyant sur les recherches d'ores et déjà existantes ${ }^{31}$. De ce

\footnotetext{
${ }^{29}$ Pour Gernot Böhme, la notion d'ambiance conduit précisément à repenser l'esthétique en ces termes. Cf. Bôhme, G. Atmosphäre. Essays zur neuen ästhetik. Frankfurt am Main, Suhrkamp, 1997.

${ }^{30}$ Telle est la position développée en autre par Hans Joas. CF. Joas, H. La créativité de l'agir. traduit de l'allemand par Pierre Rusch, Paris, Les éditions du Cerf, 1999.

${ }^{31}$ Parmi ces travaux récents traitant des ambiances architecturales et urbaines, citons l'article incontournable de Jean-François Augoyard. Cf. Augoyard, J.F. L'environnement sensible et les ambiances architecturales. L'espace Géographique. $\mathrm{n}^{\circ}$ 4, 1995, pp. 302-318. Par ailleurs, un panorama très complet de cette thématique est dressé dans une recherche récente de Pascal Amphoux. Cf. Amphoux, P. (dir.) La notion d'ambiance. Paris, éditions Plan Urbanisme Construction Architecture, 1998. Retenons aussi deux numéros spéciaux de revue publiés en 1998 : Les Cahiers de la Recherche Architecturale. « Ambiances architecturales et
} 
point de vue, une telle notion relève de paradoxes qui font à la fois sa difficulté et toute sa richesse. Cette dernière partie consistera à identifier ces paradoxes et à en tirer les conséquences en ce qui concerne nos manières d'habiter. Trois points principaux permettent de dégager la portée heuristique de l'ambiance : son caractère diffus aide à questionner l'habiter selon la catégorie du «familier », sa dimension plurisensorielle en termes de « spatialité », son versant expressif en termes d'« hospitalité ».

\section{Saisie du diffus}

De l'ordre de l'informe et du vague, l'ambiance ne se présente pas comme un objet que l'on peut facilement construire et délimiter. En remettant en cause la distinction de l'objet et du sujet, elle questionne la possibilité même de son objectivation. Si la doxa, l'opinion commune, semble particulièrement bien armée pour saisir ce domaine fluctuant et labile $e^{32}$, il en va tout autrement du discours scientifique. Mais peut être faut il opérer de biais, faire un détour, procéder de façon indirecte pour parvenir à rendre compte de cette composante essentielle de l'habiter. La question ne serait plus «qu'est-ce qu'une ambiance ?» mais « qu'est-ce qu'une ambiance permet d'être, de faire et de percevoir ? " Autrement dit, il s'agirait de poser l'ambiance en terme de potentiel, en montrant qu'elle permet de penser le passage entre de l'indifférencié et du différencié, de l'informe et de la forme ${ }^{33}$. D'une certaine manière, l'ambiance peut être considérée comme le support à partir duquel le monde sensible se configure au quotidien, comme le champ à partir duquel les phénomènes émergent et s'individuent. Il en va ici de la manière dont le monde se dote de formes mémorables et reconnaissables, lui conférant par là même un visage familier.

\section{Unité du pluriel}

Une ambiance convoque simultanément l'ensemble des sens en même temps qu'elle se spécifie à partir de chacun d'eux. Faut-il conjuguer

\footnotetext{
urbaines ", n 42/43, 1998 ; Daidalos. "Constructing Atmospheres ". $n^{\circ}$ 68, 1998. Enfin, nous ne pouvons pas manquer de faire référence à la filière doctorale "Ambiances Architecturales et Urbaines » à laquelle œuvre conjointement depuis presque dix ans le Cresson (Ecole d'Architecture de Grenoble) et le Cerma (Ecole d'Architecture de Nantes).

${ }^{32}$ C'est ce que montre très bien Anne Cauquelin dans un de ses ouvrages consacrés au « lieu commun ». Cf. Cauquelin, A. L'art du lieu commun. Paris, Seuil, 1999.

${ }^{33}$ Quelques développements très éclairants peuvent être trouvés à cet égard dans le bel ouvrage de Pierre Kaufmann. Cf. Kaufmann, P. L'expérience émotionelle de l'espace. Paris, Vrin, 1977. De son côté, Renaud Barbaras montre comment une pensée du sensible suppose d'opérer un déplacement : à une ontologie de l'objet se substitue une ontologie de l'élément . C'est ainsi que "l'élément n'est pas subjectif, il n'est pas non plus ce qui est perçu, il est la dimension selon laquelle la perception a lieu ». Cf. Barbaras, R. Le tournant de l'expérience. Paris, Vrin, 1998.
} 
l'ambiance au singulier ou au pluriel $?^{34}$ La question est loin d'être tranchée et révèle en fait les limites actuelles de nos connaissances en la matière. On peut s'appuyer sur certains résultats de la psychologie pour avancer l'idée d'une couche originaire du sentir antérieure à la division des sens ${ }^{35}$. Un tel point de vue fait valoir l'argument d'une perception amodale ou synesthésique. Néanmoins, chaque sens possède une logique spatiotemporelle spécifique et nous met en contact avec le monde d'une manière particulière. Impossible donc de dédifférencier complètement les registres sensoriels et de faire comme s'ils étaient équivalents les uns aux autres. Par contre, ne peut-on pas considérer que chacun des sens renvoie à un mode de relation préférentiel à une même ambiance $?^{36} \mathrm{Ne}$ doit on pas admettre que l'ambiance opère à chaque fois une synthèse des sens spécifique et particulière ? Tout serait alors affaire de style. Chaque manière d'être, chaque forme de motricité pourrait être lue comme une formule possible de l'unité du sensible. Dans ce cas, si l'ambiance se conjugue effectivement au pluriel, ce pluriel est moins celui des sens que celui des styles.

\section{Expression de l'ineffable}

Si l'ambiance est difficile à décrire et à mettre en mot, elle n'est pas pour autant inexprimable. Si tel était le cas, on ne pourrait pas distinguer une ambiance d'une autre et l'idée même d'ambiance n'aurait pas lieu d'être. L'ambiance est d'autant plus exprimable qu'elle se prête à des variations et des modulations, à des ruptures et des surprises. Mais encore, l'ambiance n'est pas seulement exprimable, elle est elle même expression du lieu dans lequel elle s'instaure. De ce point de vue, elle convoque des phénomènes qui la rendent plus ou moins attractive ou répulsive. Certaines ambiances donnent matière à une expérience heureuse en célébrant la possibilité de vivre ensemble et d'être à son aise. D'autres se prêtent au contraire à une expérience malheureuse en exhalant de l'hostilité et du malaise. Dans tous les cas, l'ambiance décline des qualités rythmiques qui articulent notre rapport à l'environnement et à autrui. Autrement dit, l'hospitalité d'un lieu

\footnotetext{
${ }^{34}$ La formulation de cette question revient en premier lieu à Jean-François Augoyard (op. cit. , 1995).

${ }^{35}$ Ainsi, les travaux de Daniel Stern sur le développement du nourrisson mettent en évidence l'existence de schèmes communs aux différentes modalités perceptuelles renvoyant à des "affects de vitalité". Cf. Stern, D. The Interpersonal World of the Infant: A View from Psychoanalysis and Developmental Psychology. New York, Basic Books, 1985. Selon une toute autre perspective, certains travaux récents de l'écologie de la perception remettent aussi en cause l'idée d'une séparation possible des sens. Se reporter en particulier à : Stoffregen, T. \& Bardy, B. On specification and the senses. Behavioral and Brain Sciences. Vol. XXX, 24 (1), 2001.

${ }^{36}$ Ainsi, pour Eugène Minkowski, l'ambiance « retentit » par l'auditif, « se répand » par l'olfactif, nous «touche » par le tactile, etc. Cf. Minkowski, E. [1936]Vers une cosmologie. Paris, Petite Bibliothèque Payot, 1999. De son côté, Erwin Straus montre admirablement qu'à chaque sens appartient une spatialité qui lui est propre. Cf. Straus, E. [1930] Les formes du spatial. in Figures de la subjectivité. études réunies par Jean-Jacques Courtine, Paris, éditions du CNRS, 1992, pp. 15-49.
} 
procèderait d'un double mouvement d'enveloppement et de développement : enveloppement des corps agençant des espacements variables selon des seuils et des intervalles, développement des gestes sensibilisant les interactions sociales selon des allures et des postures.

Ce bref survol des difficultés de la notion d'ambiance contribue à révéler trois dimensions essentielles de l'habiter. Chacune d'elles convoque la sensorialité d'une manière particulière et peut s'énoncer par une proposition de base.

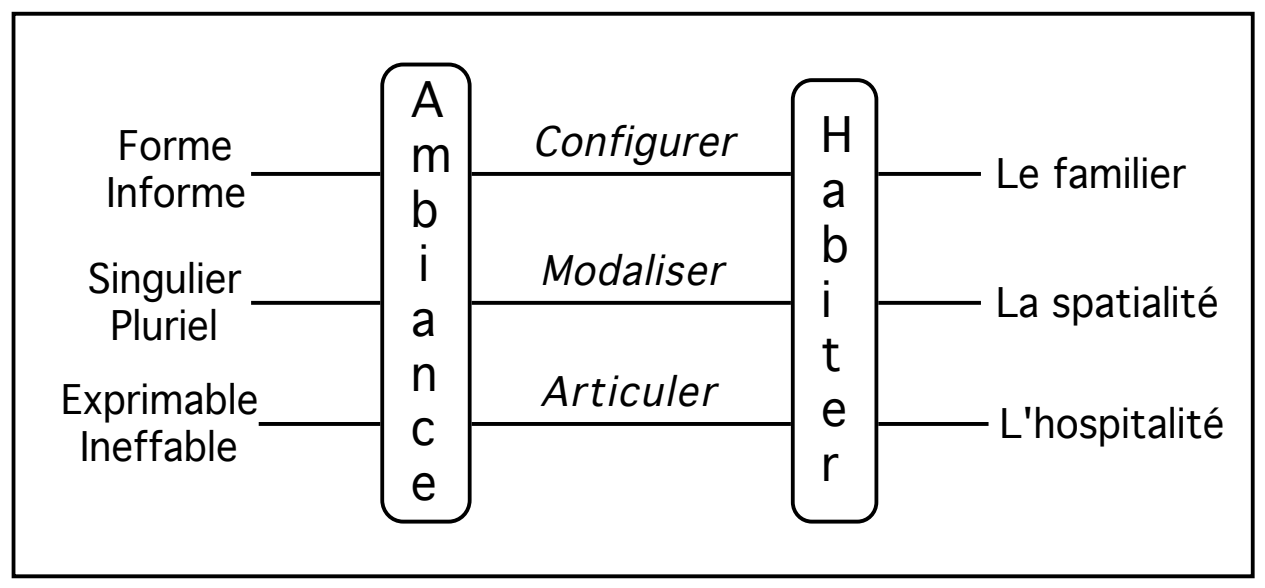

Premièrement, habiter c'est configurer. Entretenir un rapport de familiarité avec le monde suppose de donner sens à notre environnement quotidien. Un environnement m'est familier si je le reconnais et si je m'y reconnais à travers lui. L'ambiance est là pour nous rappeler qu'il nous faut pour cela le mettre en forme. Deuxièmement, habiter c'est modaliser. Investir un espace de sa présence consiste à lui donner corps en intégrant les sens dans une dynamique d'ensemble. Si la présence au monde s'éprouve à même les sens, elle se manifeste par des styles de conduites cohérents, variables selon les lieux. L'ambiance nous révèle que l'espace se décline selon une variété de manières d'être là. Troisièmement, habiter c'est articuler. Rendre un espace hospitalier engage des gestes élémentaires nous reliant les uns aux autres. Pour être accueillants, ces gestes doivent être dotés de qualités permettant une certaine souplesse et fluidité aux échanges interpersonnels. L'ambiance aide alors à mettre en évidence ce pouvoir expressif constitutif de l'être-ensemble.

\section{Conclusion}

A l'heure où la notion d'ambiance est en train de devenir un des enjeux de la recherche architecturale et urbaine, il devient urgent de clarifier cette notion autant que possible. Si toute tentative d'élaboration d'un domaine de recherche repose nécessairement sur un fond obscur qui ne peut être 
intégralement explicité ${ }^{37}$, il n'en reste pas moins possible de dégager l'état des questions et des arguments en la matière. L'émergence de cette nouvelle thématique manifeste l'intérêt croissant accordé au domaine des sens pour penser et produire l'environnement construit. Une des perspectives prometteuses de la notion d'ambiance est d'offrir la possibilité de relier et d'articuler des domaines habituellement disjoints. D'une part, à l'opposition classique entre espace vécu et espace conçu se substitue une démarche dynamique s'intéressant aux modes sensibles de structuration de l'espace et du temps. De ce point de vue, les ambiances questionnent les processus de spatialisation en œuvre à la fois dans l'expérience commune du citadin et dans l'acte de création architecturale. D'autre part, la distinction entre théorie et pratique tend à s'atténuer en faisant valoir de plein droit les savoirs et savoirs-faire des concepteurs ainsi qu'en admettant la portée opératoire des démarches scientifiques. De ce point de vue, les ambiances convoquent divers modèles d'intelligibilité du sensible rapportés à leur dimension pratique.

\footnotetext{
${ }^{37}$ Eugen Fink remarque très justement à ce propos que « dans la réflexivité la plus poussée agit toujours une immédiateté. La pensée elle-même se fonde sur ce que nous faisons sans hésitation ni réflexion. Elle a son élan productif dans l'emploi irréfléchi de ces concepts couverts d'ombres ». Cf. Fink, E. Les concepts opératoires dans la phénoménologie de Husserl. in Husserl. Cahiers de Royaumont. Philosophie n 3, Paris, Editions de Minuit, 1959, pp. 214-230.
} 


\section{Résumé :}

L'ambiance est en train de devenir un des enjeux de la recherche architecturale et urbaine. Comment et à quelles conditions une thématisation de la notion d'ambiance est-elle possible ? En quoi nous aide-t-elle à renouveler nos façons de concevoir l'habiter ? Pour répondre à de telles questions, trois arguments sont plus particulièrement explorés : 1 ) la notion d'ambiance s'inscrit dans une perspective de retour vers le concret en faisant valoir le caractère incarné et situé de l'expérience sensible, 2) elle opère un chiasme qui permet d'articuler une lecture esthétique à une lecture pragmatique de l'espace urbain, (3) elle relève de paradoxes qui interrogent le sens même de l'habiter.

\footnotetext{
Abstract :

Ambiance is in the midst of becoming one of the major issue in architectural and urban research. How and under which conditions can the concept of ambiance be defined? How can it enable us to renew the ways in which we conceive dwelling? In order to answer these questions, three points are particularly worth exploring: 1) the concept of ambiance provides a view towards a return to the concrete by emphasizing the embodiment of human experience, 2 ) it also intertwines an aesthetic approach of urban space with a pragmatic one, 3 ) and finally, it is based on paradoxes which question the very meaning of dwelling.
} 